\title{
Vorwort
}

\section{Sabine Spitzer}

Wie befreiend ist es manchmal, in eine andere Welt einzutauchen, dorthin, wo alles möglich ist, weil die herkömmlichen Regeln nicht mehr gelten, wo Vergangenheit und Zukunft zu einem Augenblick verschmelzen, wo Leben und Tod, Jugend und Alter, Hier und Dort widerspruchslos koexistieren können. Wie wertvoll ist es, den Zauber der Begegnung mit einem anderen Menschen zu spüren, über das bloße Aufeinandertreffen hinaus Verbundenheit und Verstandenwerden wahrzunehmen und als persönliche, innere Wahrheit im Herzen aufzubewahren. Wie überraschend kann es sein, im Rollentausch zum eigenen Gegenüber zu werden und plötzlich zu fühlen, was die andere fühlt, mit diesen anderen Augen zu sehen, wie man selbst in einer Szene agiert.

Wenn Grenzen überschritten werden, wenn Fantasie und Realität eine Synthese bilden, entsteht ein symbolischer Erlebensraum, der den Mehrwert an Wirklichkeit zur Entfaltung bringt - jenen Realitäts-Überschuss, für den Moreno, inspiriert von der marxistischen Terminologie seiner Zeit, den Begriff der Surplus Reality geprägt und deren hochpotente Wirksamkeit er für die Therapie nutzbar gemacht hat.

Die AutorInnen des vorliegenden Themenheftes haben es sich zur Aufgabe gemacht, die Grundgedanken zur Surplus Reality zu erläutern und zu zeigen, wie sie in den verschiedensten Bereichen damit arbeiten.

Falko von Ameln beschreibt in seinem Grundsatzartikel das Konzept der Surplus Reality als Basis des Psychodramas. Er setzt sich mit dem Realitäts-Begriff auseinander und zieht Querverbindungen zum systemischen, wie auch zum analytischen Bereich. Ergänzend bezieht er Ansätze von Goffmann und Baetson mit ein.

Christian Pajek legt seine Aufmerksamkeit auf das Verhältnis der Surplus Reality zu drei anthropologischen Ebenen der Kunst. Er zieht dazu vor allem die kunstphilosophischen Ansätze von J. W. F. Schelling aus dem 19. Jahrhundert sowie Hans Georg Gadamer und Martin Heidegger aus dem 20. Jahrhundert heran. Bezüglich der symbolischen, spielerischen und der festlichen Ebene der Kunst arbeitet er Parallelen und Überschneidungen mit der Surplus Reality heraus.

Manfred Stelzig beleuchtet die ätiologischen Zusammenhänge zwischen psychosomatischen Störungen und entwicklungspsychologischen Aspekten der Surplus Reality.

Online publiziert: 06.02 .2013

(C) Springer Fachmedien Wiesbaden 2013

S. Spitzer $(\square)$

Schwaigergasse 35/21, 1210 Wien, Österreich

E-Mail: sabine.spitzer@aon.at 
Darauf aufbauend stellt er die Arbeit mit den psychodramatischen Techniken der Surplus Reality vor, etwa die Einführung neuer, lösungsorientierter Wunschszenen als Gegensatz zu psychosomatisch krankmachenden Szenen. Dies kann durch die Darstellung der Wunschszene geschehen, durch die Entwicklung personifizierter, schützender, liebevoller Eigenschaften zu neuen Rollen und durch die Verwendung von Intermediärobjekten.

Leutz u. a. definieren Surplus Reality vorwiegend auch als Darstellung von Träumen. Diesen widmet Jutta Fürst ihren Beitrag und weist darin auf die Sinnhaftigkeit psychodramatischer Traumbearbeitung auch aus neurowissenschaftlicher Sicht hin. Sie beschreibt die Grundlagen der psychodramatischen Traumarbeit und zeigt theoretische und praktische Vorgehensweisen auf, wie die Traumebene mit anderen psychischen Ebenen in Einklang zu bringen ist.

Aus der Schweiz kommt ein Artikel über die psychodramatische Arbeit mit Kindern. Eugen Hodapp, Helena Brem und Fabian Blobel sehen im Rollen-Symbolspiel die kindgerechte Form der Surplus-Reality und zeigen, wie in diesem Erlebensraum Fantasie und Realität zusammenwachsen. Die Arbeit in der Surplus Reality umfasst die Nachgestaltung, Umgestaltung und das vollständige Verlassen der Alltagsrealität. Die GruppenleiterInnen gestalten mit den Kindern sowohl den symbolischen Handlungsraum, als auch die soziale Interaktion innerhalb der Gruppe.

Als hochwirksame Technik der Surplus Reality bezeichnete Moreno den Zauberladen. Gabriele Stiegler lädt als Ladenbesitzerin in einen solchen ein: Im Zauberladen können erwünschte Eigenschaften erworben werden, wenn dafür andere, eigene eingetauscht oder aufgegeben werden. Die neu erstandene Eigenschaft kann in der Zukunftsprobe der Surplus Reality sofort ausprobiert werden. Die Autorin stellt außerdem den Einsatz des Gruppen-Zauberladens in der Supervision vor.

Barbara Haid beschreibt in ihrem Praxisbericht die Behandlung bei Störungen der Körperwahrnehmung im Rahmen von Essstörungen. Die durch die Störung meist verdeckte Beziehung zum eigenen Körper kann durch Verkörperungstechniken auf der Ebene der Surplus Reality wieder sicht- und spürbar gemacht werden, wodurch Veränderung in kleinen Schritten möglich wird.

Märchen und Mythen enthalten in symbolisierter und verdichteter Form wichtige, ganzheitliche Aspekte menschlicher Konfliktlösungen. Die nächsten beiden Artikeln widmen sich diesen Themen: Regina Bulian erzählt vom Mythos „Amor und Psyche“ und wie dessen Surplus Reality die psychodramatischen GruppenspielerInnen zu neuen Erfahrungen und Perspektiven führt.

Reinhard Krüger erläutert die Technik des Bewältigungsmärchens, mit der sich frühe, unerfüllte Sehnsüchte stillen lassen. In der Surplus Reality des Schreibens erleben die PatientInnen sich und die Geschichte ihrer Kindheit neu. Die therapeutische Arbeit mit Bewältigungsmärchen ist primär eine Distanzierungstechnik, ermöglicht in weiterer Folge den Zugang zu verdeckten Teilen des Selbst und fördert mit Hilfe der Surplus Reality die Fähigkeit zur seelischen Konfliktbearbeitung.

In seiner prosaischen Schilderung einer Burschengruppe lässt uns Karl Staudinger intensiv in die psychodramatische Spielwelt eintauchen. Gemeinsam mit Toni Samtstachel, Däumling und Nino Angstlos zeigt er auf, wie die gemeinsamen symbolischen Realitäten zu Rollenerweiterung und positiver Entwicklung genutzt werden können. 
In der Rubrik „Der andere Artikel“ untersucht Norbert Engelbrecht Parallelen und Unterschiede zwischen psychodramatischem und personzentrierten Ansatz. Er erläutert die personzentrierten Schlüsselbegriffe Empathie, Kongruenz und Akzeptanz sowie die wesentlichsten Strömungen der klassischen, dialogischen und der EncountergruppenArbeit. Dabei werden Ähnlichkeiten in den Grundstrukturen der beiden therapeutischen Schulen erkennbar.

Ein dickes Buch, Heiligenbildchen als Souvenirs, ein Paket Toleranz - und einen Bericht hat Verena Dudde aus Indien mitgebracht. Als Psychodramatikerin und Sozialanthropologin erzählt sie von ihrer Suche nach dem Psychodrama auf dem Subkontinent.

Gute Nachrichten kommen aus der Schweiz: Die Weiterbildung in Humanistischer Psychotherapie mit Schwerpunkt Psychodrama wurde von der Schweizerischen Gesellschaft für Psychiatrie und Psychotherapie (SGPP) anerkannt. Roger Schaller stellt in seinem Forums-Beitrag das Institut für Psychodrama und Aktionsmethoden (ipda) und den Weiterbildungslehrgang vor.

Wir bedanken uns bei den AutorInnen sehr herzlich für ihre Mitarbeit an diesem Heft und wünschen den LeserInnen eine interessante Surplus-Lektüre!

Sabine Spitzer und Christian Pajek 